\title{
BIOELECTRONICS
}

\section{Buckled scalable intracellular bioprobes}

\author{
Controllably buckling an array of vertical field effect transistors enables intracellular electrophysiological mapping \\ with high spatial and temporal resolutions.
}

\section{Woohyun Park and Chi Hwan Lee}

$\mathrm{T}$ he ability to directly measure intracellular electrophysiology in electrogenic cells has provided abundant insights into the biophysical functions of nerves, muscles, and synapses, which is thereby crucial for neurophysiological, cardiological, and pharmacological research ${ }^{1}$. The patch-clamp technique that involves inserting a glass micropipette into the interior of individual cells has been the gold standard for high-precision intracellular recording. However, the patch-clamp technique is highly invasive and unsuitable for multiplexing and parallelization (typically $\leq 10$ simultaneous measurements), limiting its utility in many applications due to low testing throughput. Impressive improvement has been made in miniaturizing the glass micropipette into a nanopipette (that is, $15-30 \mathrm{~nm}$ in the tip diameter) to reduce the invasiveness, but the testing throughput still remains limited by the difficulty of keeping a large quantity of nanopipettes in the cells ${ }^{2}$. In the past decade, multidisciplinary efforts to combine nanotechnologies have enabled rapid success in producing alternative solutions using either passive or active bioprobes at the cellular and subcellular length scales ${ }^{3-5}$. A prominent example of the passive bioprobes involves using a total of 4,096 multiplexed platinum black nanoelectrodes to simultaneously record action potential propagations from thousands of connected neurons (that is, high spatial resolution $)^{6}$. Another example of the active bioprobes involves using a number $(\leq 10)$ of nanoscale field effect transistors (FETs) at the tip of curved silicon nanowires that can spontaneously penetrate the cell membranes and then capture subthreshold and low-amplitude intracellular signals (that is, high temporal resolution) ${ }^{7}$. Despite great advances, current approaches exhibit a trade-off between spatial and temporal scales in the multiplexed intracellular recording.

Writing in Nature Nanotechnology, Yue Gu et al. now integrate a scalable array of FETs into a bioprobe, enabling the multiplexed three-dimensional (3D) mapping of intracellular electrophysiological signals

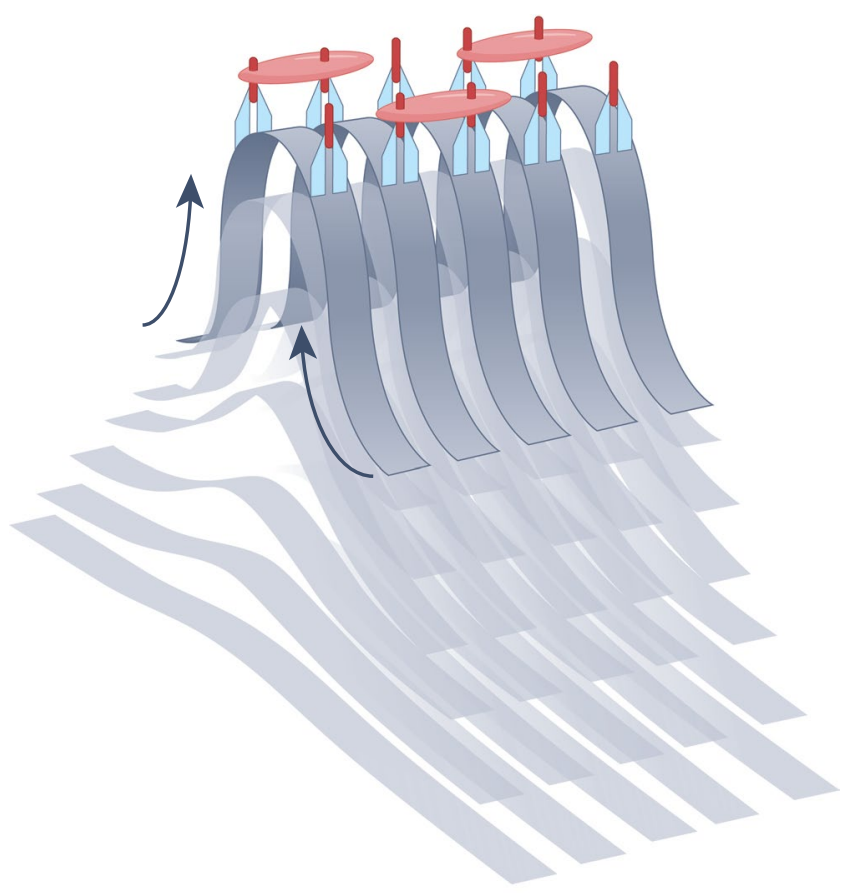

Fig. 1 | Controlled buckling process to pop up bioprobes for 3D intracellular recording. Schematic illustration of the geometric transformation of the pre-fabricated planar FETs into a 3D pop-up structure by controlled compressive buckling for intracellular recording. Credit: figure adapted with permission from ref. ${ }^{8}$, Springer Nature Ltd.

and their conduction paths with high spatial and temporal resolutions ${ }^{8}$. The authors employ a compressive buckling technique that allows the pre-fabricated planar FETs to be geometrically transformed into a 3D pop-up structure in a controlled fashion through the guidance of foldable hinges ${ }^{9}$ (Fig. 1). The resulting bioprobe exhibits a vertically ordered array of FETs that can penetrate through the cell membrane with minimal invasiveness and also record the transmembrane potentials with high fidelity. To further reduce the invasiveness, the surface of the probe tips can be functionalized with phospholipid bilayers to form tight intracellular junctions and smooth internalization. The bioprobe supports the faithful recording of subthreshold action potentials (that is, cell membrane oscillations of 5-15 mV) from cardiomyocytes with high sensitivity-to-noise ratios and temporal resolution comparable to the gold standard patch-clamp technique. The bioprobe is also capable of monitoring the dynamic change in the beating rhythm, resting membrane potential, and action potential duration of cardiomyocytes in the presence of ion channel blocking drugs (for example, nifedipine and tetrodotoxin), highlighting its potential utility in pharmaceutical screening. Uniquely, the bioprobe is scalable in overall size and configuration to better interface with the curvilinear surface of large tissues, thereby enhancing the recording quality and testing throughput. For instance, the authors tailor the bioprobe to deploy a total of 128 FETs within a 3D constructed cardiac tissue at different heights (that is, 48 FETs each at 20 and $40 \mu \mathrm{m}, 32$ FETs at $60 \mu \mathrm{m}$ ) in a uniformly distributed manner. The resulting 
bioprobe is able to capture the intracellular action potentials of the cardiac tissue and also reveal the signal conduction paths in $3 \mathrm{D}$. Leveraging the high spatial and temporal resolutions of the bioprobe, the authors successfully measure the intracellular signal conduction velocity of a cardiomyocyte (that is, $0.182 \mathrm{~m} \mathrm{~s}^{-1}$ ) and a cardiac tissue (that is, $0.0188 \pm 0.0075 \mathrm{~m} \mathrm{~s}^{-1}$ ).

In principle, the bioprobe could be more scalable to adopt most cells and maybe even smaller subcellular compartments through fine refinement of the tip size, spacing, and relative positions. Therefore, the intracellular information recorded from the scaled bioprobes would be useful for better understanding of subcellular electrophysiology, organellar ionic dynamics, organelle-cell membrane interaction, and electrical coupling between different cells. Beyond the applications presented by the authors, it may also be interesting to further scale up the bioprobe toward interfacing with human 3D organoids that closely mimic their corresponding in vivo organs. In this context, the resulting bioprobe could be useful in allowing the minimally invasive penetration of multiple FETs through the organoid membrane for real-time intra-organoid recording in a conceptually similar manner to recently reported 3D mesostructures ${ }^{10}$. In the era of a global pandemic, one could also envision the use of the intra-organoid recording for evaluating the unknown effects of clinical antiviral drugs for COVID-19, such as Kevzara and Actemra, on a specific organoid of interest. In addition, these bioprobes, which are built on a stretchable soft elastomer, may permit their use in in vivo recording settings on animal studies in a manner that can reduce the invasiveness and also ensure the recording fidelity against tissue movements. Although several technical obstacles remain in the bioprobe and other similar nanosensors for improving long-term stability in intracellular recording, the study by Yue Gu et al., encompassing 3D pop-up nanostructures and their implementation into a deeper understanding of intracellular responses, represents a noticeable leap.
Woohyun Park (D)1 and Chi Hwan Lee (D) 1,2凶 ${ }^{1}$ School of Mechanical Engineering, Purdue University, West Lafayette, IN, USA. ${ }^{2}$ Weldon School of Biomedical Engineering, School of Mechanical Engineering, School of Materials Engineering, and Birck Nanotechnology Center, Purdue University, West Lafayette, IN, USA.

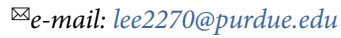

Published online: 7 March 2022

https://doi.org/10.1038/s41565-021-01028-6

\section{References}

1. Jiang, Y. \& Tian, B. Nat. Rev. Mater. 3, 473-490 (2018).

2. Jayant, K. et al. Nat. Nanotechnol. 12, 335-342 (2017).

3. Hong, G. \& Lieber, C. M. Nat. Rev. Neurosci. 20, 330-345 (2019).

4. Abbott, J. et al. Nat. Nanotechnol. 12, 460-466 (2017).

5. Robinson, J. T. et al. Nat. Nanotechnol. 7, 180-184 (2012).

6. Abbott, J. et al. Nat. Biomed. Eng. 4, 232-241 (2020).

7. Zhao, Y. et al. Nat. Nanotechnol. 14, 783-790 (2019).

8. Gu, Y. et al. Nat. Nanotechnol. https://doi.org/10.1038/s41565021-01040-w (2021).

9. Xu, S. et al. Science 347, 154-159 (2015).

10. Ryu, H. et al. Adv. Mater. 33, 2100026 (2021).

\section{Competing interests}

The authors declare no competing interests.

\title{
NUCLEIC ACID DELIVERY
}

\section{Nucleic acid delivery differences across species}

\author{
Examining nucleic acid delivery in humanized animal models might accelerate nanoparticle optimization and \\ potentially improve clinical translation strategies.
}

\section{Omar F. Khan}

A dvances such as large-scale combinatorial chemistry and high-throughput genetic data analysis have allowed researchers to create vast numbers of unique nanoparticles that deliver therapeutic nucleic acids like messenger ribonucleic acid (mRNA). Each nanoparticle has the potential to cure disease and improve life quality; however, we remain limited in our ability to predict which candidate will work best in humans. Animal models such as mice and non-human primates have been the standard for preclinical testing; however, with marked differences in anatomy, cell structure and even immune system function, animals are not always the best predictor of human success. Now, writing in Nature Nanotechnology, Hatit, Lokugamage and Dobrowolski ${ }^{1}$ report a hybrid approach: mice with livers that contain not only mouse cells but also human or primate liver cells. With this model, the team tested the delivery and uptake of nucleic acids via a variety of nanoparticles, and directly compared differences between a liver's mouse cells and its human or primate cells. The result is a comprehensive study that demonstrates how differences between species can be preserved and observed using this hybrid liver approach, which may allow researchers to better predict human efficacy well before human trials.

One of the greatest challenges to translating a nucleic acid nanoparticle technology is optimization. Although many nanoparticles begin their development using a human cell culture in high-throughput screenings for efficacy, these conditions lack the context of the human body with its myriad of cells and complex network of interconnected tissues and biochemical processes. Although cell-based assays excel at evaluating many subcellular events, the impact caused by the non-trivial journey from the extracellular space to the intracellular one is missing. Proteins quickly adsorb to nanoparticles ${ }^{2}$. Afterwards, they can drain into the lymphatic system, or be cleared via the kidney, liver and spleen. Between administration and clearance, there is a limited opportunity for nanoparticle uptake by other cells and tissues. Screening and optimization in animals maximize this opportunity.

Animal tests serve multiple purposes, including biodistribution, toxicity and efficacy studies, but ultimately, the marked differences in anatomy and immune system cell composition ${ }^{3}$ means that, at best, animal testing is an informative compromise. To justify a human clinical trial, nanoparticles are optimized in progressively higher orders 